Rückenschmerzen

\section{Reden statt Röntgen}

— Bei $60-80 \%$ der Patienten haben Rückenschmerzen keine organische Ursache, erinnert die Deutsche Gesellschaft für Unfallchirurgie. Die Nationale Versorgungsleitlinie Kreuzschmerz empfiehlt deshalb, erst dann eine bildgebende Untersuchung wie Röntgen oder MRT anzuordnen, wenn der Schmerz auch nach vier bis sechs Wochen nicht abklingt, die körperliche Aktivität einschränkt oder zunimmt.

In über $90 \%$ der Fälle wird diesen Vorgaben auch entsprochen und auf eine nicht indizierte oder zu frühe radiologische Bildgebungsdiagnostik verzichtet. Das ergab eine Analyse von Versichertendaten gesetzlicher Krankenversicherungen. „Im Vordergrund einer guten Diagnostik bei Rückenbeschwerden steht die fachkundige Befragung des Patienten und eine sachgerechte körperliche Untersuchung", wird Professor Bernd Kladny, Generalsekretär der Deutschen Gesellschaft für Orthopädie und Orthopädische Chirurgie, in der Mitteilung zitiert. Bei der Erstuntersuchung gehe es vor allem darum, Warnzeichen zu erkennen, die etwa auf einen Wirbelbruch, einen Bandscheibenvorfall mit Nervenschaden oder

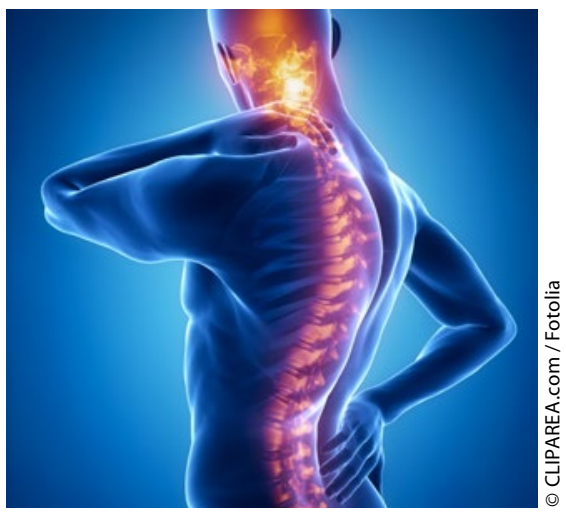

eine Entzündung hindeuten. „Lassen sich keine entsprechenden Hinweise feststellen, kann man bei erstmaligen akuten Schmerzen in den ersten vier Wochen zunächst das Symptom Schmerz behandeln und den Patienten ausführlich aufklären", so Kladny. In diesen Fällen helfen akut Schmerzmittel sowie Bewegung im Alltag und gezielte Übungen. Bei $80 \%$ aller Rückenpatienten klingen die Schmerzen innerhalb von wenigen Tagen oder Wochen wieder ab. "Tritt nach vier bis sechs Wochen bei anhaltenden aktivitätseinschränkenden oder zunehmenden Kreuzschmerzen keine Besserung ein, ist es angeraten, den Einsatz von bildgebenden Verfahren zu überprüfen", räumt Kladny ein.

(eb)
Paracetamol

\section{Pränatale Exposition mit Einfluss aufs Kind?}

— Bei der Studie „Avon Longitudinal Study of Parents and Children" (ALSPAC) handelt es sich um eine prospektive Geburtskohortenstudie, an der zwischen April 1991 und Dezember 1992 insgesamt 7.796 Mütter mit ihren Kindern und Partnern teilgenommen hatten [Stergiakouli E et al. JAMA Pediatr 2016; 170:964-70]. Die Befragung zum Paracetamolgebrauch erfolgte während der Schwangerschaft in der 18. und 32. Woche sowie im Alter der Kinder von 61 Monaten. Mithilfe des „Strengths and Difficulties Questionnaire" (SDQ) wurden die Mütter zu Verhaltensstörungen ihrer Kinder im Alter von sieben Jahren befragt. Wie die Wissenschaftler um Dr. Evie Stergiakouli vom Medical Research Council in Bristol berichten, nahmen 4.415 Mütter (53\%) Paracetamol in der 18. Schwangerschaftswoche (SSW) ein, 3.381 Mütter in der 32. SSW. Den Berechnungen zufolge war die Wahrscheinlichkeit für Verhaltensstörungen durch diese Selbstbehandlung während der Schwangerschaft deutlich um etwa das Eineinhalbfache (Risk Ratio [RR]:1,42; 95\%-KI 1,25-1,62) erhöht. Die Risikoerhöhung galt auch für Hyperaktivitätssymptome (RR: 1,31; 95\%-KI 1,16-1,49) sowie für emotionale Symptome (RR: 1,29; 95\%-KI 1,09-1,53). Die RR-Werte lagen jeweils bei Paracetamolanwendung in der 32. SSW höher als in der 18. SSW.

Aus den Studienergebnissen lässt sich kein kausaler Zusammenhang zwischen der Paracetamolanwendung während der Schwangerschaft und der Entwicklung von Verhaltensstörungen ableiten. Zumal von den Studienteilnehmerinnen keine Angaben zur Indikation der Paracetamoltherapie sowie zur Dosierung der Arznei und zur Dauer der Behandlung gemacht wurden. In den Fachinformationen zur Anwendung von Paracetamol wird unter anderem darauf hingewiesen, dass Paracetamol während der Schwangerschaft bei klinischer Notwendigkeit eingenommen werden darf. Es sollte jedoch „in der geringsten wirksamen Dosis über einen möglichst kurzen Zeitraum und so selten wie möglich eingenommen werden".

(ple)

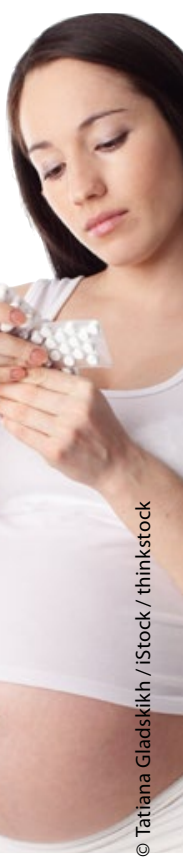

